\title{
Lax Triples for Integrable Surfaces in Three-Dimensional Space
}

\author{
Jan L. Cieśliński ${ }^{1}$ and Artur Kobus ${ }^{2}$ \\ ${ }^{1}$ Uniwersytet w Białymstoku, Wydział Fizyki, Ulica Ciołkowskiego 1L, 15-245 Białystok, Poland \\ ${ }^{2}$ Politechnika Białostocka, Wydział Budownictwa i Inżynierii Środowiska, Ulica Wiejska 45E, 15-351 Białystok, Poland \\ Correspondence should be addressed to Jan L.Cieśliński; j.cieslinski@uwb.edu.pl
}

Received 24 April 2016; Accepted 28 June 2016

Academic Editor: Boris G. Konopelchenko

Copyright (C) 2016 J. L. Cieśliński and A. Kobus. This is an open access article distributed under the Creative Commons Attribution License, which permits unrestricted use, distribution, and reproduction in any medium, provided the original work is properly cited.

We study Lax triples (i.e., Lax representations consisting of three linear equations) associated with families of surfaces immersed in three-dimensional Euclidean space $\mathbb{E}^{3}$. We begin with a natural integrable deformation of the principal chiral model. Then, we show that all deformations linear in the spectral parameter $\lambda$ are trivial unless we admit Lax representations in a larger space. We present an explicit example of triply orthogonal systems with Lax representation in the group Spin(6). Finally, the obtained results are interpreted in the context of the soliton surfaces approach.

\section{Introduction}

We consider geometric problems associated with Lax triples, that is, Lax representations consisting of three linear equations with a spectral parameter. They can be interpreted as integrable deformations of problems corresponding to one of the involved Lax pairs. The problem of finding integrable deformations of surfaces in $\mathbb{E}^{3}$ is an interesting and nontrivial task. There are just few papers on that subject; see, for example, [1-3]. However, we point out that the central result of [1], namely, Theorem 3.2, is wrong. This theorem claims that for any augmented system of Gauss-Mainardi-Codazzi equations there exists an explicit Lax representation with the spectral parameter: system (3.6) in [1]. Unfortunately, one can easily check that the spectral parameter can be easily eliminated from this system by performing simple algebraic calculations.

In this paper we suggest another methodology. We start from Lax representations of prescribed form in order to obtain special cases of integrable systems. We checked two cases: Lax representations with three different simple poles (an integrable deformation of the principal chiral model; see Section 2) and Lax representations linear in the spectral parameter. All $S U(2)$-valued Lax representations linear in $\lambda$ turned out to be trivial; see Section 3. However, there exists a nontrivial Lax representation in a larger space for a special class of orthogonal nets in $\mathbb{E}^{3}[4]$; see also Section 4. Finally, in Section 5, we shortly present more general context for studying integrable differential geometry: soliton surfaces approach [5] and Lie point symmetries for introducing the spectral parameter [6,7].

Throughout this paper we usually use the Lie group $S U(2)$ instead of $S O(3)$, taking into account the isomorphism of corresponding Lie algebras: $s o(3) \simeq s u(2)$. We can assume, for instance,

$$
\left(\begin{array}{ccc}
0 & -a_{3} & -a_{2} \\
a_{3} & 0 & a_{1} \\
a_{2} & -a_{1} & 0
\end{array}\right) \longleftrightarrow-\frac{i}{2}\left(\begin{array}{cc}
a_{3} & a_{1}-i a_{2} \\
a_{1}+i a_{2} & -a_{3}
\end{array}\right) .
$$

$S U(2)$ is double covering of $S O(3)$, so all our results can be projected on $S O(3)$ when necessary. In Section 4 we make use of another isomorphism, $s u(2) \simeq \operatorname{spin}(3)$. Actually, also $S U(2) \simeq \operatorname{Spin}(3)$.

\section{An Integrable Deformation of the Principal Chiral Model}

The principal chiral model is defined by the following system of two equations [8-10]:

$$
\begin{aligned}
& A_{t}=\frac{1}{2}[B, A], \\
& B_{x}=\frac{1}{2}[A, B],
\end{aligned}
$$


where $A, B$ are elements of a Lie algebra $g$. Equations (2) are equivalent to

$$
\begin{aligned}
A_{t}-B_{x}+[A, B] & =0, \\
A_{t}+B_{x} & =0 .
\end{aligned}
$$

The chiral model has the Lax representation

$$
\begin{aligned}
& \Psi_{x}=\frac{A}{1-\lambda} \Psi, \\
& \Psi_{t}=\frac{B}{1+\lambda} \Psi,
\end{aligned}
$$

where $\Psi=\Psi(x, t ; \lambda) \in G$ ( $G$ is the corresponding Lie group). The chiral model is integrable in the sense of the theory of solitons. In particular, Darboux transformation and multisoliton solutions are known [10]. Denoting $\Phi=$ $\Psi(x, t ; 0)$ we can express $A$ and $B$ in terms of $\Phi$ :

$$
\begin{gathered}
A=\Phi_{x} \Phi^{-1}, \\
B=\Phi_{t} \Phi^{-1} .
\end{gathered}
$$

Then the first equation of (3) is identically satisfied and system (3) reduces to a single equation for $\Phi$ :

$$
\left(\Phi_{x} \Phi^{-1}\right)_{t}+\left(\Phi_{t} \Phi^{-1}\right)_{x}=0
$$

By changing variables $\xi=(1 / 2)(x+t), \eta=(i / 2)(t-x)$, we transform (6) into

$$
\left(\Phi_{\xi} \Phi^{-1}\right)_{\xi}+\left(\Phi_{\eta} \Phi^{-1}\right)_{\eta}=0
$$

Solutions to this equation are harmonic maps from $\mathbb{R}^{2}$ into $G$ provided that $\xi, \eta$ are real $[9,11]$.

We propose an extension of the principal chiral model which is derived from the following Lax representation:

$$
\begin{aligned}
& \Psi_{x}=\frac{A}{1-\lambda} \Psi, \\
& \Psi_{t}=\frac{B}{1+\lambda} \Psi, \\
& \Psi_{y}=\frac{\kappa C}{\kappa-\lambda} \Psi,
\end{aligned}
$$

where $\kappa \in \mathbb{R}$ (we assume $|\kappa| \neq 1$ ), $\Psi=\Psi(x, t, y ; \lambda)$ takes values in a Lie group $G$, and $A, B, C$ belong to the corresponding Lie algebra $g$. In particular, we can take $G=$ $S U(2)$. This case yields an integrable deformation of surfaces in $\mathbb{E}^{3}$. Compatibility conditions for $(8)$ read

$$
\begin{aligned}
A_{t} & =\frac{1}{2}[B, A], \\
B_{y} & =\frac{\kappa}{1+\kappa}[C, B], \\
C_{x} & =\frac{1}{1-\kappa}[A, C],
\end{aligned}
$$

$$
\begin{aligned}
B_{x} & =\frac{1}{2}[A, B], \\
C_{t} & =\frac{1}{1+\kappa}[B, C], \\
A_{y} & =\frac{\kappa}{1-\kappa}[A, C] .
\end{aligned}
$$

Denoting $\Phi=\Psi(x, t, y ; 0)$ we have, similarly to the chiral model case,

$$
\begin{aligned}
& A=\Phi_{x} \Phi^{-1} \\
& B=\Phi_{t} \Phi^{-1} \\
& C=\Phi_{y} \Phi^{-1} .
\end{aligned}
$$

Then, compatibility conditions (9) reduce to three equations for one function $\Phi$ :

$$
\begin{gathered}
\left(\Phi_{x} \Phi^{-1}\right)_{t}+\left(\Phi_{t} \Phi^{-1}\right)_{x}=0 \\
\left(\Phi_{x} \Phi^{-1}\right)_{y}-\kappa\left(\Phi_{y} \Phi^{-1}\right)_{x}=0 \\
\left(\Phi_{t} \Phi^{-1}\right)_{y}+\kappa\left(\Phi_{y} \Phi^{-1}\right)_{t}=0
\end{gathered}
$$

Proposition 1. Any solution $\Phi_{0}(x, t)$ of the chiral model (6) admits an extension (unique up to translations in $y$ ) to a solution $\Phi$ of the deformed chiral model (11) such that $\Phi\left(x, t, y_{0}\right)=\widehat{\Phi}_{0}(x, t)$ for some $y_{0}$.

Proof. System (11) is equivalent to

$$
\begin{array}{r}
\left(\Phi_{x} \Phi^{-1}\right)_{t}+\left(\Phi_{t} \Phi^{-1}\right)_{x}=0 \\
(1-\kappa) \Phi_{x y}-\Phi_{x} \Phi^{-1} \Phi_{y}+\kappa \Phi_{y} \Phi^{-1} \Phi_{x}=0 \\
(1+\kappa) \Phi_{t y}-\Phi_{t} \Phi^{-1} \Phi_{y}-\kappa \Phi_{y} \Phi^{-1} \Phi_{t}=0
\end{array}
$$

The last two equations of (12) can be rewritten as a linear system for one unknown $\Phi_{y}$ :

$$
\begin{aligned}
\frac{\partial}{\partial x} \Phi_{y} & =\frac{A \Phi_{y}-\kappa \Phi_{y} \widehat{A}}{1-\kappa} \\
\frac{\partial}{\partial t} \Phi_{y} & =\frac{B \Phi_{y}+\kappa \Phi_{y} \widehat{B}}{1+\kappa},
\end{aligned}
$$

where $\widehat{A}=\Phi^{-1} A \Phi$ and $\widehat{B}=\Phi^{-1} B \Phi$. Therefore, let us consider linear system

$$
\begin{aligned}
& \frac{\partial \Gamma}{\partial x}=\frac{A \Gamma-\kappa \Gamma \widehat{A}}{1-\kappa} \\
& \frac{\partial \Gamma}{\partial t}=\frac{B \Gamma+\kappa \Gamma \widehat{B}}{1+\kappa} .
\end{aligned}
$$

We will show that the compatibility conditions for (14) are equivalent to the first equation of (12) which, in turn, is 
equivalent to (3). Compatibility conditions $\Phi_{y x}=\Phi_{x y}$ can be written as

$$
P \Phi_{y}-\Phi_{y} Q=0
$$

where

$$
\begin{aligned}
P & =(1+\kappa) A_{t}+A B-(1-\kappa) B_{x}-B A, \\
Q & =\kappa(1+\kappa) \widehat{A}_{t}+\kappa^{2} \widehat{B} \widehat{A}-\kappa^{2} \widehat{A} \widehat{B}+\kappa(1-\kappa) \widehat{B}_{x} .
\end{aligned}
$$

We immediately see that (3) imply $P=0$. In order to show $Q=0$ we compute

$$
\begin{aligned}
\widehat{A}_{t} & =\Phi^{-1}\left(A_{t}+[A, B]\right) \Phi, \\
\widehat{B}_{x} & =\Phi^{-1}\left(B_{x}+[B, A]\right) \Phi, \\
{[\widehat{A}, \widehat{B}] } & =\Phi^{-1}[A, B] \Phi .
\end{aligned}
$$

Therefore,

$$
\begin{aligned}
\widehat{A}_{t}-\widehat{B}_{x}-[\widehat{A}, \widehat{B}] & =\Phi^{-1}\left(A_{t}-B_{x}+[A, B]\right) \Phi=0, \\
\widehat{A}_{t}+\widehat{B}_{x} & =\Phi^{-1}\left(A_{t}+B_{x}\right) \Phi=0
\end{aligned}
$$

where we once more took into account (3). Equations (18) imply $Q=0$.

Thus, given a solution $\Phi_{0}$ of (6) we obtain a unique solution $\Gamma_{0}$ of linear system (14). Then, we define $\Phi\left(x, t, y_{0}\right):=$ $\Phi_{0}(x, t)$ and interpret $\Gamma_{0}(x, y)$ as $y$-derivative of $\Phi(x, t, y)$ at $y=y_{0}$.

We proceed to showing the integrability of deformed chiral model (11) by constructing the Darboux-Bäcklund transformation, following [12]. In what follows we usually suppress the dependence on $x, t, y$, sometimes also the dependence on $\lambda$. We will write, for instance, $D(\lambda)$ or $D$ instead of $D(x, t, y ; \lambda)$.

Lemma 2. The Darboux transformation $\widetilde{\Psi}=D \Psi$ with a canonical normalization (i.e., $D(\infty)=I$ ) applied to a linear problem $\Psi_{x}=U(\lambda) \Psi$ preserves the constraint $U(\infty)=0$.

Proof. We have $\widetilde{U}=D_{x} D^{-1}+D U D^{-1}$. Therefore, the canonical normalization implies $\widetilde{U}(\infty)=U(\infty)$.

Proposition 3. The Darboux transformation for $G L(n)$ valued linear problem (8) is given by

$$
\widetilde{\Psi}(\lambda)=f(\lambda)\left(I+\frac{\lambda_{1}-\mu_{1}}{\lambda-\lambda_{1}} P\right) \Psi(\lambda),
$$

where $f(\lambda)$ is a constant (i.e., it does not depend on $x, t, y$ ), $\lambda_{1}$ and $\mu_{1}$ are complex parameters, and $P$ is uniquely defined by its kernel $\operatorname{ker} P=\Psi\left(\lambda_{1}\right) V_{\mathrm{ker}}$ and image $\operatorname{Im} P=\Psi\left(\mu_{1}\right) V_{\mathrm{Im}}$, where $V_{\mathrm{ker}}$ and $V_{\mathrm{Im}}$ are subspaces of $\mathbb{C}^{n}$ such that $V_{\mathrm{ker}} \oplus V_{\mathrm{Im}}=$ $\mathbb{C}^{n}$ and $\Psi(\lambda)$ is a solution of (8) assumed to be known. The corresponding transformation for the chiral field $\Phi$ reads

$$
\widetilde{\Phi}=f_{0}\left(I+\frac{\mu_{1}-\lambda_{1}}{\lambda_{1}} P\right) \Phi,
$$

where $f_{0} \equiv f(0)$ is a constant. The reduction $\operatorname{SL}(n, C)$ consists in choosing

$$
f(\lambda)=\left(\frac{\lambda-\lambda_{1}}{\lambda-\mu_{1}}\right)^{d / n}
$$

where $d=\operatorname{dim} \operatorname{Im} P$. The reduction $U(n)$ consists in adding the following constraints: $\mu_{1}=\bar{\lambda}_{1}$ and $V_{\mathrm{Im}}$ is orthogonal to $V_{\mathrm{ker}}$.

Proof. Formula (19) preserves all poles of the linear problem (8), as shown, for instance, in [12]. There is also another condition to be preserved: all matrices of our linear problem vanish at $\lambda=\infty$. By Lemma 2 this constraint is preserved by any Darboux matrix with the canonical normalization. Formula (20) follows from (19) by substitution $\lambda=0$. In particular, we have $\Phi=\Psi(0)$. Implications of the reductions $U(n)$ and $S L(n, C)$ are described in [12] in detail.

Proposition 3 proves integrability of the related nonlinear system. All steps of the proof are exactly the same as in the case of the principal chiral model. Therefore, multisoliton solution can be obtained in the standard way; see $[10,13]$.

\section{SU(2)-Valued Lax Triples Linear in $\lambda$ Are Trivial}

Let us consider $S U(2)$-valued Lax representations linear in $\lambda$ :

$$
\begin{aligned}
& \Psi_{x}=(A+\lambda L) \Psi, \\
& \Psi_{t}=(B+\lambda M) \Psi, \\
& \Psi_{y}=(C+\lambda N) \Psi,
\end{aligned}
$$

where $A, B, C, L, M, N$ are $s u(2)$-valued functions of $x$, $t, y$ (without dependence on $\lambda$ ) and $\Psi=\Psi(x, t, y ; \lambda) \epsilon$ $S U(2)$. Subscripts $x, t, y$ denote differentiation. Compatibility conditions $\left(\Psi_{x t}=\Psi_{t x}, \Psi_{t y}=\Psi_{y t}, \Psi_{x y}=\Psi_{y x}\right) \mathrm{read}$

$$
\begin{gathered}
{[L, M]=0,} \\
{[L, N]=0,} \\
{[M, N]=0,} \\
A_{t}-B_{x}+[A, B]=0, \\
B_{y}-C_{t}+[B, C]=0, \\
C_{x}-A_{y}+[C, A]=0, \\
L_{t}-M_{x}+[L, B]+[A, M]=0, \\
M_{y}-N_{t}+[M, C]+[B, N]=0, \\
N_{x}-L_{y}+[N, A]+[C, L] .
\end{gathered}
$$

We make use of isomorphism $\mathbb{E}^{3} \simeq s u(2)$, where by $\mathbb{E}^{3}$ we mean the three-dimensional Euclidean space with scalar and skew product. In this isomorphism the scalar product is identified with the Killing-Cartan form (without entering into details we denote by $\mathbf{e}_{1}, \mathbf{e}_{2}, \mathbf{e}_{3}$ one of the orthonormal 
frames) and the skew product with the Lie bracket (commutator of matrices). In particular, vanishing commutator of two elements means that they are parallel and so forth.

The first set of compatibility conditions, namely, (23), can be easily solved: $L, M, N$ are parallel. Therefore, there exists a unit matrix $m \in s u(2)$ and real functions $\alpha, \beta, \gamma$ such that

$$
\begin{aligned}
L & =\alpha m, \\
M & =\beta m, \\
N & =\gamma m .
\end{aligned}
$$

Any unit element $m \in s u(2)$ can be obtained from any constant unit element, say $\mathbf{e}_{3}$, by a rotation. In other words, there exists $T \in S U(2)$ such that

$$
m=T^{-1} \mathbf{e}_{3} T
$$

Substituting (26) into (25) we get

$$
\begin{gathered}
\alpha_{t} m+\alpha m_{t}-\beta_{x} m-\beta m_{x}+\alpha[m, B]+\beta[A, m]=0, \\
\beta_{y} m+\beta m_{y}-\gamma_{t} m-\gamma m_{t}+\beta[m, C]+\gamma[B, m]=0, \\
\gamma_{x} m+\gamma m_{x}-\alpha_{y} m-\alpha m_{y}+\gamma[m, A]+\alpha[C, m]=0 .
\end{gathered}
$$

Taking into account that any $[m, X]$ is orthogonal to $m$ (for any $X$ ) and any derivative of $m$ is orthogonal to $m$ (because $m$ is a unit element), we can decompose (28) into parallel and orthogonal parts. In particular, we have

$$
\begin{aligned}
& \alpha_{t}=\beta_{x}, \\
& \beta_{y}=\gamma_{t}, \\
& \gamma_{x}=\alpha_{y} .
\end{aligned}
$$

Hence, there exists a real function $\varphi=\varphi(x, t, y)$ such that

$$
\begin{aligned}
& \alpha=\varphi_{x}, \\
& \beta=\varphi_{t}, \\
& \gamma=\varphi_{y} .
\end{aligned}
$$

Formula (27) suggests gauge transformation $\widehat{\Psi}=T \Psi$. Then, using also (26) and (30), we have

$$
\begin{gathered}
\widehat{\Psi}_{x}=\left(\widehat{A}+\lambda \varphi_{x} \mathbf{e}_{3}\right) \widehat{\Psi}, \\
\widehat{\Psi}_{t}=\left(\widehat{B}+\lambda \varphi_{t} \mathbf{e}_{3}\right) \widehat{\Psi}, \\
\widehat{\Psi}_{y}=\left(\widehat{C}+\lambda \varphi_{y} \mathbf{e}_{3}\right) \widehat{\Psi},
\end{gathered}
$$

where

$$
\begin{aligned}
& \widehat{A}=T_{x} T^{-1}+T A T^{-1}, \\
& \widehat{B}=T_{t} T^{-1}+T B T^{-1}, \\
& \widehat{C}=T_{y} T^{-1}+T C T^{-1} .
\end{aligned}
$$

Therefore, all $S U(2)$-valued Lax representations linear in $\lambda$ can be reduced to (31). It turns out that this system can be reduced further. Matrices $\widehat{A}, \widehat{B}, \widehat{C}$ satisfy compatibility conditions consisting of (24) and

$$
\begin{aligned}
{\left[\mathbf{e}_{3}, \varphi_{t} \widehat{A}-\varphi_{x} \widehat{B}\right] } & =0, \\
{\left[\mathbf{e}_{3}, \varphi_{x} \widehat{C}-\varphi_{y} \widehat{A}\right] } & =0, \\
{\left[\mathbf{e}_{3}, \varphi_{t} \widehat{C}-\varphi_{y} \widehat{B}\right] } & =0
\end{aligned}
$$

Hence, after similar consideration to above, we obtain

$$
\begin{aligned}
& \widehat{A}=\varphi_{x} \widehat{Q}+a \mathbf{e}_{3}, \\
& \widehat{B}=\varphi_{t} \widehat{Q}+c \mathbf{e}_{3}, \\
& \widehat{C}=\varphi_{y} \widehat{Q}+c \mathbf{e}_{3},
\end{aligned}
$$

where $\widehat{Q}$ is a matrix-valued function orthogonal to $\mathbf{e}_{3}$ and $a$, $b, c$ are real functions. From (24) we get

$$
\begin{aligned}
& a_{t} \mathbf{e}_{3}-b_{x} \mathbf{e}_{3}+\varphi_{x} \widehat{Q}_{t}-\varphi_{t} \widehat{Q}_{x}+\varphi_{x} b\left[\widehat{Q}, \mathbf{e}_{3}\right] \\
& +a \varphi_{t}\left[\mathbf{e}_{3}, \widehat{Q}\right]=0, \\
& b_{y} \mathbf{e}_{3}-c_{t} \mathbf{e}_{3}+\varphi_{t} \widehat{Q}_{y}-\varphi_{y} \widehat{Q}_{t}+\varphi_{t} c\left[\widehat{Q}, \mathbf{e}_{3}\right] \\
& \quad+b \varphi_{y}\left[\mathbf{e}_{3}, \widehat{Q}\right]=0, \\
& c_{x} \mathbf{e}_{3}-a_{y} \mathbf{e}_{3}+\varphi_{y} \widehat{Q}_{x}-\varphi_{x} \widehat{Q}_{y}+\varphi_{y} a\left[\widehat{Q}, \mathbf{e}_{3}\right] \\
& \quad+c \varphi_{x}\left[\mathbf{e}_{3}, \widehat{Q}\right]=0 .
\end{aligned}
$$

The components parallel to $\mathbf{e}_{3}$ yield

$$
\begin{aligned}
& a_{t}=b_{x}, \\
& b_{y}=c_{t}, \\
& c_{y}=a_{y},
\end{aligned}
$$

which means that there exists a real function $\chi$ such that

$$
\begin{aligned}
& a=\chi_{x}, \\
& b=\chi_{t}, \\
& c=\chi_{y} .
\end{aligned}
$$

The function $\chi$ can be eliminated by another gauge transformation:

$$
\Phi=e^{-\chi \mathbf{e}_{3}} \widehat{\Psi}
$$

Then, we obtain the following linear problem:

$$
\begin{gathered}
\Phi_{x}=\varphi_{x}\left(\lambda \mathbf{e}_{3}+Q\right) \Phi, \\
\Phi_{t}=\varphi_{t}\left(\lambda \mathbf{e}_{3}+Q\right) \Phi, \\
\Phi_{y}=\varphi_{y}\left(\lambda \mathbf{e}_{3}+Q\right) \Phi,
\end{gathered}
$$


where $Q=e^{-\chi \mathbf{e}_{3}} \widehat{Q} e^{\chi \mathbf{e}_{3}}$. The obtained linear problem is trivial because it can be transformed into a single equation by a change of variables. Indeed, taking $\tilde{x}=\varphi(x, y, t), \widetilde{t}=t$, and $\tilde{y}=y$ we transform system (39) into

$$
\begin{aligned}
\Phi_{\tilde{x}} & =\left(\lambda \mathbf{e}_{3}+Q\right) \Phi, \\
\Phi_{\tilde{t}} & =0, \\
\Phi_{\tilde{y}} & =0 .
\end{aligned}
$$

Therefore $Q$ can be taken as an arbitrary function of $\tilde{x}$. No differential equations are involved.

\section{An Integrable System Associated with Triply Orthogonal Coordinates}

Because of isomorphism (1) $S U(2)$-valued Lax representations are a rich source of integrable geometries in $\mathbb{E}^{3}$; see [5]. In some problems, however, $S U(2)$ assumption is too restrictive. For instance, searching for integrable geometries associated with Lax representations linear in the spectral parameter we obtained a negative result: there are no nontrivial $S U$ (2)-cases (see Section 3). In order to show integrability of geometric problems in $\mathbb{E}^{3}$ one sometimes needs a larger Lie group; see [14-16].

Here we present in more detail the case of a special system of triply orthogonal coordinates. We consider the following Lax representation:

$$
\begin{aligned}
& \Psi_{x}=\frac{1}{2}\left(\mathbf{a}_{1} \lambda+\mathbf{b}_{1}\right) \mathbf{e}_{1} \Psi, \\
& \Psi_{t}=\frac{1}{2}\left(\mathbf{a}_{2} \lambda+\mathbf{b}_{2}\right) \mathbf{e}_{2} \Psi, \\
& \Psi_{y}=\frac{1}{2}\left(\mathbf{a}_{3} \lambda+\mathbf{b}_{3}\right) \mathbf{e}_{3} \Psi,
\end{aligned}
$$

where subscripts $x, t, y$ denote differentiation and

$$
\begin{aligned}
& \mathbf{a}_{1}=\alpha_{11} \mathbf{e}_{4}+\alpha_{21} \mathbf{e}_{5}+\alpha_{31} \mathbf{e}_{6}, \\
& \mathbf{b}_{1}=\beta_{21} \mathbf{e}_{2}+\beta_{31} \mathbf{e}_{3}, \\
& \mathbf{a}_{2}=\alpha_{12} \mathbf{e}_{4}+\alpha_{22} \mathbf{e}_{5}+\alpha_{32} \mathbf{e}_{6}, \\
& \mathbf{b}_{2}=\beta_{12} \mathbf{e}_{1}+\beta_{32} \mathbf{e}_{3}, \\
& \mathbf{a}_{3}=\alpha_{13} \mathbf{e}_{4}+\alpha_{23} \mathbf{e}_{5}+\alpha_{33} \mathbf{e}_{6}, \\
& \mathbf{b}_{3}=\beta_{13} \mathbf{e}_{1}+\beta_{23} \mathbf{e}_{2},
\end{aligned}
$$

$\alpha_{j k}, \beta_{j k}$ are real functions, and $\mathbf{e}_{k}$ are generators of the Clifford algebra $\mathscr{C}_{6,0}$; that is, they satisfy the following relations:

$$
\begin{aligned}
\mathbf{e}_{k}^{2} & =1, \quad(\text { for } k=1,2, \ldots, 6), \\
\mathbf{e}_{j} \mathbf{e}_{k} & =-\mathbf{e}_{k} \mathbf{e}_{j}, \quad(\text { for } j \neq k) .
\end{aligned}
$$

Note that the Lie algebra of linear problem (41) is spanned by bivectors $\mathbf{e}_{j} \mathbf{e}_{k}$ which means that this is Spin(6)-valued (double covering of corresponding $S O(6)$ linear problem). Compatibility conditions for (41) read

$$
\begin{aligned}
\left\langle\mathbf{a}_{j} \mid \mathbf{a}_{k}\right\rangle & =0, \quad(\text { for } j \neq k), \\
\partial_{j} \alpha_{i k} & =\beta_{j k} \alpha_{i j}, \quad(\text { for } j \neq k), \\
\partial_{j} \beta_{i k} & =\beta_{i j} \beta_{j k}, \quad(\text { for } i \neq j \neq k \neq i), \\
\partial_{k} \beta_{k j}+\partial_{j} \beta_{j k}+\beta_{i k} \beta_{i j} & =0, \quad(\text { for } i \neq j \neq k \neq i),
\end{aligned}
$$

where $\partial_{1} \equiv \partial_{x}, \partial_{2}=\partial_{t}, \partial_{3}=\partial_{y}$, and

$$
\left\langle\mathbf{a}_{j} \mid \mathbf{a}_{k}\right\rangle:=\sum_{i=1}^{3} \alpha_{i j} \alpha_{i k} .
$$

From (44) it follows that $\left\langle\mathbf{a}_{1} \mid \mathbf{a}_{1}\right\rangle,\left\langle\mathbf{a}_{2} \mid \mathbf{a}_{2}\right\rangle,\left\langle\mathbf{a}_{3} \mid \mathbf{a}_{3}\right\rangle$ are functions of one variable (resp., $x, t$, or $y$ ). These functions can be eliminated by a change of variables. Therefore without loss of the generality we can assume

$$
\left\langle\mathbf{a}_{1} \mid \mathbf{a}_{1}\right\rangle=\left\langle\mathbf{a}_{2} \mid \mathbf{a}_{2}\right\rangle=\left\langle\mathbf{a}_{3} \mid \mathbf{a}_{3}\right\rangle=1 .
$$

Solutions of the nonlinear system (44) and (45) have a direct connection with the geometry of triply orthogonal systems in $\mathbb{E}^{3}$. Namely, the metric

$$
d s^{2}=H_{1}^{2} d x^{2}+H_{2}^{2} d t^{2}+H_{3}^{2} d y^{2}
$$

describes a triply orthogonal system of coordinates if and only if Lamé coefficients $H_{1}, H_{2}, H_{3}$ satisfy the Lamé equations $[17,18]$ :

$$
\begin{gathered}
\frac{\partial^{2} H_{1}}{\partial y \partial t}=\frac{1}{H_{2}} \frac{\partial H_{2}}{\partial y} \frac{\partial H_{1}}{\partial t}+\frac{1}{H_{3}} \frac{\partial H_{3}}{\partial t} \frac{\partial H_{1}}{\partial y}, \\
\frac{\partial^{2} H_{2}}{\partial x \partial y}=\frac{1}{H_{3}} \frac{\partial H_{3}}{\partial x} \frac{\partial H_{2}}{\partial y}+\frac{1}{H_{1}} \frac{\partial H_{1}}{\partial y} \frac{\partial H_{2}}{\partial x}, \\
\frac{\partial^{2} H_{3}}{\partial t \partial x}=\frac{1}{H_{1}} \frac{\partial H_{1}}{\partial t} \frac{\partial H_{3}}{\partial x}+\frac{1}{H_{2}} \frac{\partial H_{2}}{\partial x} \frac{\partial H_{3}}{\partial t}, \\
\frac{\partial}{\partial x}\left(\frac{1}{H_{1}} \frac{\partial H_{2}}{\partial x}\right)+\frac{\partial}{\partial t}\left(\frac{1}{H_{2}} \frac{\partial H_{1}}{\partial t}\right)+\frac{1}{H_{3}^{2}} \frac{\partial H_{1}}{\partial y} \frac{\partial H_{2}}{\partial y}=0, \\
\frac{\partial}{\partial y}\left(\frac{1}{H_{2}} \frac{\partial H_{3}}{\partial t}\right)+\frac{\partial}{\partial y}\left(\frac{1}{H_{3}} \frac{\partial H_{2}}{\partial y}\right)+\frac{1}{H_{1}^{2}} \frac{\partial H_{2}}{\partial x} \frac{\partial H_{3}}{\partial x}=0 \\
\left.\frac{1}{H_{1}} \frac{\partial H_{3}}{\partial x}\right)+\frac{1}{H_{2}^{2}} \frac{\partial H_{3}}{\partial t} \frac{\partial H_{1}}{\partial t}=0 .
\end{gathered}
$$

Defining the so called rotation coefficients [18]

$$
\beta_{k j}=\frac{\partial_{k} H_{j}}{H_{k}}
$$

one can show that system (45) is equivalent to (49).

The whole system of compatibility conditions, (44), (45), and (47), is equivalent to the Lamé equations (49) with the following constraint:

$$
H_{1}^{2}+H_{2}^{2}+H_{3}^{2}=1 .
$$


It is sufficient to define (for any fixed $j$ )

$$
H_{k}=\alpha_{j k}
$$

Then, condition (51) follows from the orthogonality of the matrix $\alpha_{j k}$. The Lamé system with constraint (51) was first considered by Darboux; see [17, book III, chapter X]. Modern approach to the Darboux transformations for this system can be found in $[15,19,20]$ (in [20] the constraint (51) is not explicitly presented). Some other reductions of Lamé equations are discussed in [21]. Lamé equations without this constraint are also integrable; see $[18,22]$ and references quoted therein.

Throughout this section we used a convenient language of Clifford numbers. It is worthwhile noticing that system (41) evaluated at $\lambda=0$ becomes equivalent to

$$
\begin{gathered}
\Psi_{x}=A \Psi, \\
\Psi_{t}=B \Psi, \\
\Psi_{y}=C \Psi,
\end{gathered}
$$

where

$$
\begin{aligned}
& A=\left(\begin{array}{ccc}
0 & -\beta_{21} & -\beta_{31} \\
\beta_{21} & 0 & 0 \\
\beta_{31} & 0 & 0
\end{array}\right) \longleftrightarrow \frac{1}{2} \beta_{21} \mathbf{e}_{2} \mathbf{e}_{1}+\frac{1}{2} \beta_{31} \mathbf{e}_{3} \mathbf{e}_{1}, \\
& B=\left(\begin{array}{ccc}
0 & \beta_{12} & 0 \\
-\beta_{12} & 0 & -\beta_{32} \\
0 & \beta_{32} & 0
\end{array}\right) \longleftrightarrow \frac{1}{2} \beta_{12} \mathbf{e}_{1} \mathbf{e}_{2}+\frac{1}{2} \beta_{32} \mathbf{e}_{3} \mathbf{e}_{2}, \\
& C=\left(\begin{array}{ccc}
0 & 0 & \beta_{13} \\
0 & 0 & \beta_{23} \\
-\beta_{13} & -\beta_{23} & 0
\end{array}\right) \longleftrightarrow \frac{1}{2} \beta_{13} \mathbf{e}_{1} \mathbf{e}_{3}+\frac{1}{2} \beta_{23} \mathbf{e}_{2} \mathbf{e}_{3} .
\end{aligned}
$$

One can easily check that elements $(1 / 2) \mathbf{e}_{2} \mathbf{e}_{3},(1 / 2) \mathbf{e}_{3} \mathbf{e}_{1}$, and $(1 / 2) \mathbf{e}_{1} \mathbf{e}_{2}$ generate the Lie algebra $s u(2)$; compare (1). Compatibility conditions for (53) yield augmented GaussMainardi-Codazzi system [1].

\section{Soliton Surfaces}

Soliton surfaces approach $[5,23]$ (see also [24]) can be easily applied to the analysis of deformation of surfaces in $\mathbb{E}^{3}$. Namely, having an SU(2)-valued Lax representation

$$
\begin{aligned}
& \Psi_{x}=U(\lambda) \Psi, \\
& \Psi_{t}=V(\lambda) \Psi, \\
& \Psi_{y}=W(\lambda) \Psi,
\end{aligned}
$$

such that $U(0)=A, V(0)=B, W(0)=C$, we define

$$
\mathbf{r}=\left.\Psi^{-1} \Psi_{\lambda}\right|_{\lambda=0}
$$

This is $y$-family of surfaces in $s u(2) \simeq \mathbb{E}^{3}$. Soliton surfaces can be easily analysed on the implicit level (fundamental forms), which is one of the important advantages of this approach. Indeed, we compute

$$
\begin{aligned}
\mathbf{r}_{x} & =\Psi^{-1} U_{\lambda} \Psi, \\
\mathbf{r}_{t} & =\Psi^{-1} V_{\lambda} \Psi, \\
\mathbf{r}_{y} & =\Psi^{-1} W_{\lambda} \Psi, \\
\mathbf{r}_{x t} & =\Psi^{-1}\left(U_{\lambda t}+\left[U_{\lambda}, B\right]\right) \Psi, \text { etc. }
\end{aligned}
$$

Therefore, in any particular case all fundamental forms can be calculated without the explicit knowledge of $\Psi$. Geometric interpretation often helps to understand trivial or degenerated cases. For linear problem (22) we get

$$
\begin{aligned}
& \mathbf{r}_{x}=\Psi^{-1} L \Psi, \\
& \mathbf{r}_{t}=\Psi^{-1} M \Psi, \\
& \mathbf{r}_{y}=\Psi^{-1} N \Psi .
\end{aligned}
$$

Therefore (23) simply mean that all tangent vectors are parallel. Hence, system (22) describes a curve rather than a family of deformed surfaces.

In the case of triply orthogonal systems with constraint (51) the soliton surfaces approach shows an unexpected feature: soliton submanifold (56) is immersed in a sixdimensional space. However, by making an appropriate projections on three-dimensional spaces we obtain three different triply orthogonal systems of coordinates in $\mathbb{E}^{3}[15]$, mutually related by the so called Combescure transformations.

Another advantage of this approach is a unification of several integrable models on the same soliton surface [25]. For instance, in the case of chiral model (4) we easily obtain

$$
\begin{aligned}
\mathbf{r}_{x} & =\Phi^{-1} A \Phi, \\
\mathbf{r}_{t} & =\Phi^{-1} B \Phi, \\
\mathbf{r}_{x t} & =\Phi^{-1}\left(A_{t}+[A, B]\right) \Phi .
\end{aligned}
$$

Hence, taking into account (2), we obtain

$$
\mathbf{r}_{x t}=\frac{1}{2}\left[\mathbf{r}_{x}, \mathbf{r}_{t}\right]
$$

Thus the soliton surface of the principal chiral model is swept out by the motion of a relativistic string model [26]. Similar computation for other pairs of variables yields

$$
\begin{aligned}
& \mathbf{r}_{x y}=\frac{\kappa}{1-\kappa}\left[\mathbf{r}_{x}, \mathbf{r}_{y}\right], \\
& \mathbf{r}_{y t}=\frac{\kappa}{1+\kappa}\left[\mathbf{r}_{y}, \mathbf{r}_{t}\right] ;
\end{aligned}
$$

that is, we get relativistic strings, as well.

\section{Further Developments}

Soliton surfaces approach was generalized on semisimple Lie groups [5] and $\operatorname{Spin}(p, q)$ groups [4, 15]. There are also further 
developments with more stress on symmetries [24, 27, 28]. Lie point symmetries turned out to be very promising as a tool for inserting the spectral parameter into Gauss-Weingarten equations ("nonparametric linear problems") associated with surfaces immersed in $\mathbb{E}^{3}[6,7,29-32]$. In other words, it turns out that in some cases the spectral parameter $\lambda$ is a group parameter.

A natural next step consists in finding some integrable cases by studying Lie point symmetries for special systems of augmented Gauss-Mainardi-Codazzi equations (53). We hope to get new integrable cases. The work in this direction is in progress.

\section{Competing Interests}

The authors declare that they have no competing interests.

\section{Acknowledgments}

The research is supported by Polish Ministry of Science and Higher Education. In particular, the second author (Artur Kobus) realizes his research in the framework of the Project S/WBiIŚ/3/2016.

\section{References}

[1] P. Bracken, "Deformation of surfaces in three-dimensional space induced by means of integrable systems," Nonlinear Analysis: Real World Applications, vol. 14, no. 3, pp. 1331-1339, 2013.

[2] B. G. Konopelchenko, "Nets in $R^{3}$, their integrable evolutions and the DS hierarchy," Physics Letters A, vol. 183, no. 2-3, pp. 153-159, 1993.

[3] B. G. Konopelchenko and U. Pinkall, "Integrable deformations of affine surfaces via the Nizhnik-Veselov-Novikov equation," Physics Letters A, vol. 245, no. 3-4, pp. 239-245, 1998.

[4] J. L. Cieśliński, "A class of linear spectral problems in Clifford algebras," Physics Letters A, vol. 267, no. 4, pp. 251-255, 2000.

[5] A. Sym, "Soliton surfaces and their applications," in Geometric Aspects of the Einstein Equations and Integrable Systems, R. Martini, Ed., vol. 239 of Lecture Notes in Physics, pp. 154-231, Springer, Berlin, Germany, 1985.

[6] J. L. Cieśliński, P. Goldstein, and A. Sym, "On integrability of the inhomogeneous Heisenberg ferromagnet model: examination of a new test," Journal of Physics A: Mathematical and General, vol. 27, no. 5, pp. 1645-1664, 1994.

[7] J. Krasil'shchik and M. Marvan, "Coverings and integrability of the Gauss-Mainardi-Codazzi equations," Acta Applicandae Mathematicae, vol. 56, no. 2-3, pp. 217-230, 1999.

[8] M. Mañas, "The principal chiral model as an integrable system," in Harmonic Maps and Integrable Systems, A. P. Fordy and J. C. Wood, Eds., pp. 147-173, Vieweg, Braunschweig, Germany, 1994.

[9] K. Uhlenbeck, "Harmonic maps into Lie groups: classical solutions of the chiral model," Journal of Differential Geometry, vol. 30, no. 1, pp. 1-50, 1989.

[10] V. E. Zakharov and A. V. Mikhailov, "On the integrability of classical spinor models in two-dimensional space-time," Communications in Mathematical Physics, vol. 74, no. 1, pp. 2140, 1980 .
[11] M. A. Guest, Harmonic Maps, Lie Groups and Integrable Systems, Cambridge University Press, 1997.

[12] J. Cieśliński, "An algebraic method to construct the Darboux matrix," Journal of Mathematical Physics, vol. 36, no. 10, pp. 5670-5706, 1995.

[13] J. L. Cieśliński, "Algebraic construction of the Darboux matrix revisited," Journal of Physics A: Mathematical and Theoretical, vol. 42, no. 40, Article ID 404003, 40 pages, 2009.

[14] J. Cieśliński, "The Darboux-Bianchi transformation for isothermic surfaces. Classical results versus the soliton approach," Differential Geometry and Its Applications, vol. 7, no. 1, pp. 1-28, 1997.

[15] J. L. Cieśliński, "Geometry of submanifolds derived from spin-valued spectral problems," Theoretical and Mathematical Physics, vol. 137, no. 1, pp. 1396-1405, 2003.

[16] J. Cieśliński, P. Goldstein, and A. Sym, "Isothermic surfaces in $\mathbf{E}^{3}$ as soliton surfaces," Physics Letters A, vol. 205, no. 1, pp. 3743, 1995.

[17] G. Darboux, Leçons sur les Systèmes Orthogonaux et les Coordonnées Curvilignes, Gauthier-Villars, Paris, France, 2nd edition, 1910.

[18] V. E. Zakharov, "Description of the $n$-orthogonal curvilinear coordinate systems and Hamiltonian integrable systems of hydrodynamic type, I: integration of the Lamé equations," Duke Mathematical Journal, vol. 94, no. 1, pp. 103-139, 1998.

[19] W. Biernacki and J. L. Cieśliński, "A compact form of the Darboux-Bäcklund transformation for some spectral problems in Clifford algebras," Physics Letters A, vol. 288, no. 3-4, pp. 167$172,2001$.

[20] J. Cieśliński, “The cross ratio and Clifford algebras," Advances in Applied Clifford Algebras, vol. 7, no. 2, pp. 133-139, 1997.

[21] O. I. Mokhov, "Lax pairs for equations describing compatible nonlocal poisson brackets of hydrodynamic type and integrable reductions of the Lam equations," Theoretical and Mathematical Physics, vol. 138, no. 2, pp. 238-249, 2004.

[22] L. V. Bogdanov and B. G. Konopelchenko, "Lattice and $q$ difference Darboux-Zakharov-Manakov systems via $\bar{\partial}$-dressing method," Journal of Physics A: Mathematical and General, vol. 28, pp. L173-L178, 1995.

[23] A. Sym, "Soliton surfaces," Lettere Al Nuovo Cimento, vol. 33, no. 12, pp. 394-400, 1982.

[24] J. Cieśliński, "A generalized formula for integrable classes of surfaces in Lie algebras," Journal of Mathematical Physics, vol. 38, no. 8, pp. 4255-4272, 1997.

[25] A. Sym, "Soliton surfaces II. Geometric unification of solvable nonlinearities," Lettere Al Nuovo Cimento, vol. 36, no. 10, pp. 307-312, 1983.

[26] A. Sym, O. Ragnisco, D. Levi, and M. Bruschi, "Soliton surfaces VII. Relativistic string in external field: general integral and particular solutions," Lettere al Nuovo Cimento, vol. 44, no. 8, pp. 529-536, 1985.

[27] A. S. Fokas and I. M. Gelfand, "Surfaces on Lie groups, on Lie algebras, and their integrability," Communications in Mathematical Physics, vol. 177, no. 1, pp. 203-220, 1996.

[28] A. M. Grundland, S. Post, and D. Riglioni, "Soliton surfaces and generalized symmetries of integrable systems," Journal of Physics A: Mathematical and Theoretical, vol. 47, no. 1, Article ID 015201, 14 pages, 2014.

[29] H. Baran and M. Marvan, "Classification of integrable Weingarten surfaces possessing an $s l(2)$-valued zero curvature representation," Nonlinearity, vol. 23, pp. 2577-2597, 2010. 
[30] J. Cieśliński, "Lie symmetries as a tool to isolate integrable geometries," in Nonlinear Evolution Equations and Dynamical Systems (Proceedings NEEDS '91), M. Boiti, L. Martina, and F. Pempinelli, Eds., pp. 260-268, World Scientific, Singapore, 1992.

[31] J. Cieśliński, "Non-local symmetries and a working algorithm to isolate integrable geometries," Journal of Physics A: Mathematical and General, vol. 26, no. 5, pp. L267-L271, 1993.

[32] M. Marvan, "On the spectral parameter problem," Acta Applicandae Mathematicae, vol. 109, no. 1, pp. 239-255, 2010. 


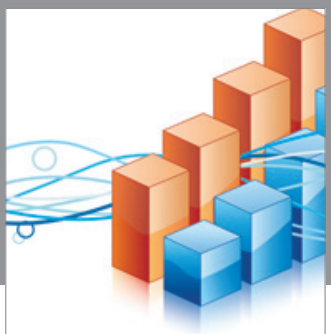

Advances in

Operations Research

vatem alat4

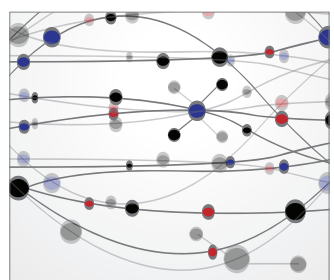

\section{The Scientific} World Journal
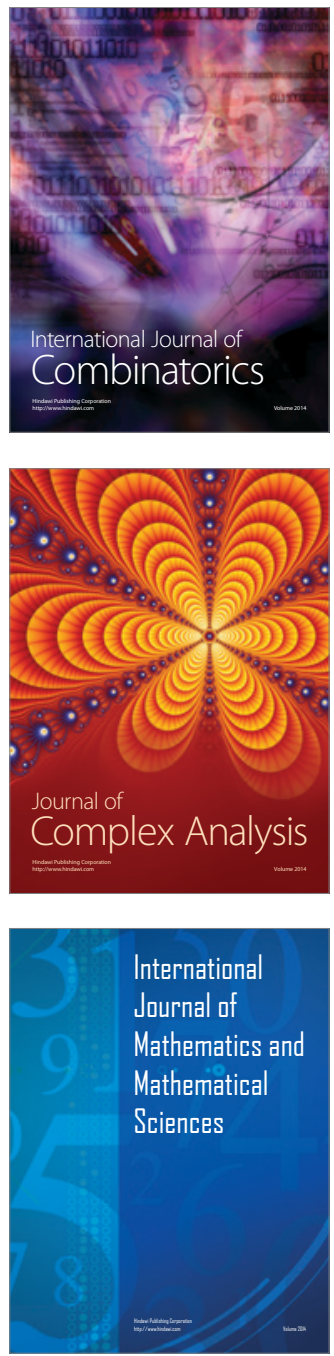
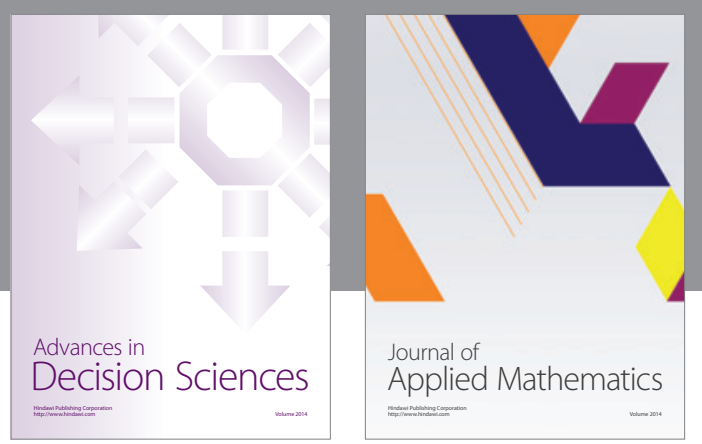

Algebra

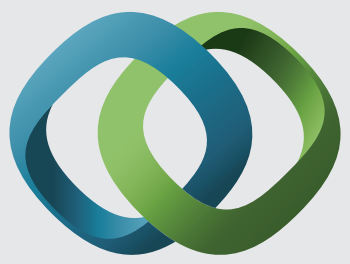

\section{Hindawi}

Submit your manuscripts at

http://www.hindawi.com
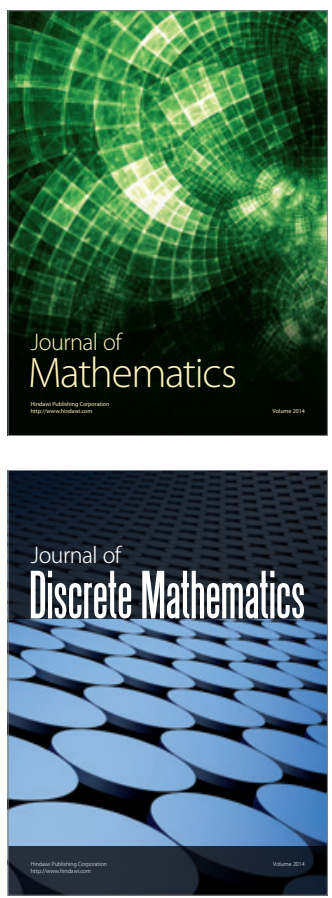

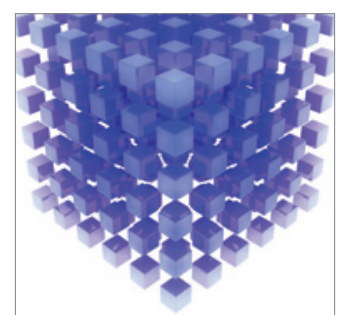

Mathematical Problems in Engineering
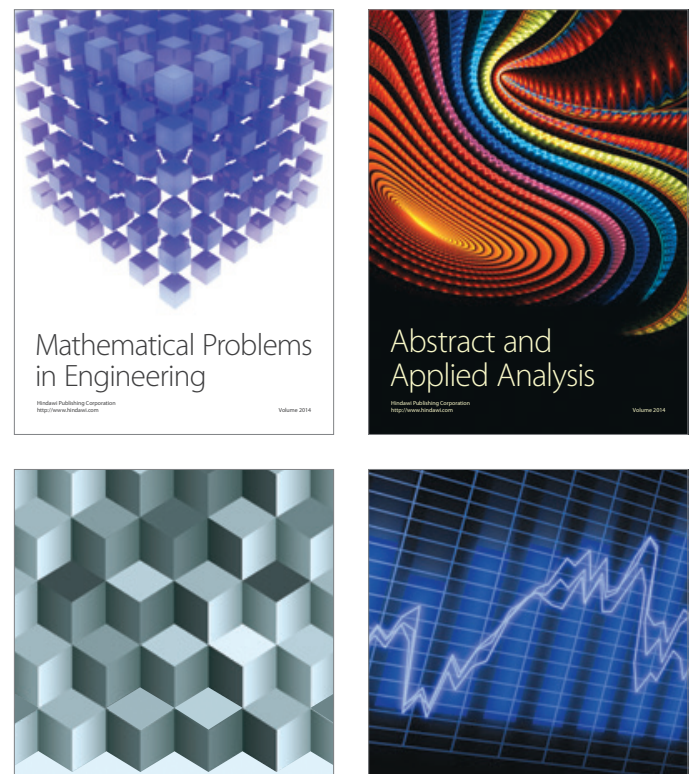

Journal of

Function Spaces

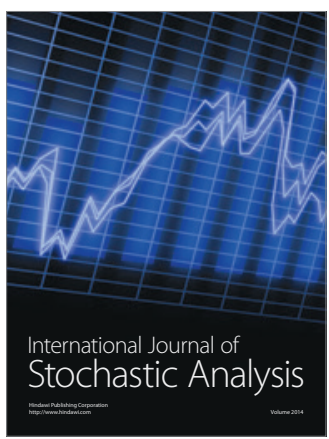

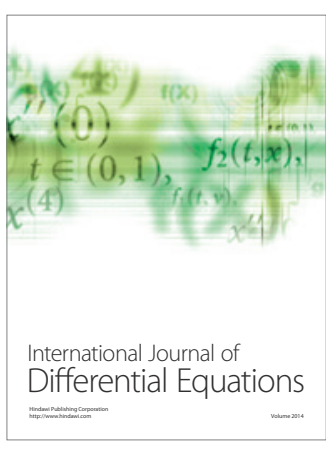
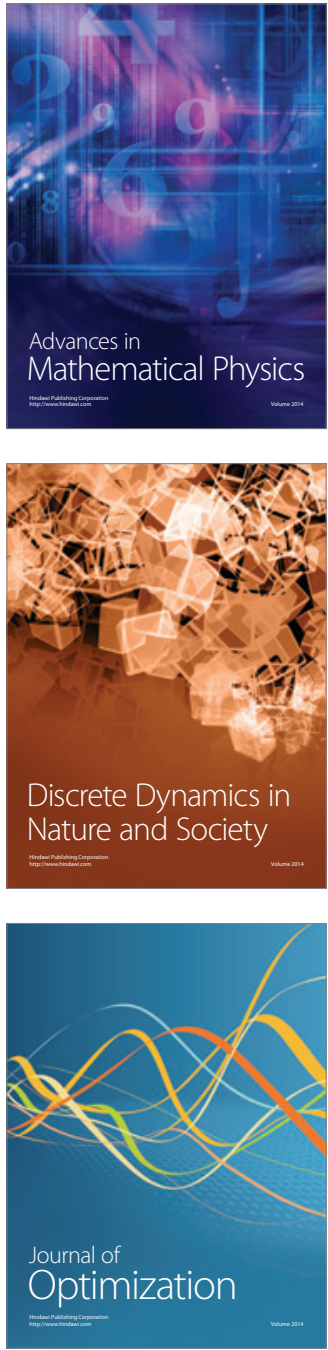